\title{
Clumping in stellar winds and interiors
}

\section{Götz Gräfener}

\author{
Argelander Institut für Astronomie, Universität Bonn, \\ Auf dem Hügel 71, 53121 Bonn, Germany \\ email: goetz@astro.uni-bonn.de
}

\begin{abstract}
The uncertain clumping properties pose a major problem for the quantitative analysis and the modelling of hot star winds. New results suggest that also the outer envelopes of massive stars may be affected by clumping, with important consequences for their observable radii and ionising properties. In this talk I will discuss how clumping is incorporated in stellar interior and wind/atmosphere models, how current theoretical results compare with observations, and what we can learn from a combination of stellar interior models and winds.
\end{abstract}

\section{Introduction}

Over the last twenty years it became clear that the radiatively-driven winds of hot massive stars are structured and inhomogeneous. This is in stark contrast to stellar wind and atmosphere models, where these outflows are often treated as homogeneous. The presence of inhomogeneities has a strong influence on the opacities and the radiative transfer in stellar winds. It thus affects the modelling and diagnostics of stellar winds, and introduces large uncertainties in theoretical predictions and empirical determinations of the mass-loss rates of massive stars.

More recent works started to investigate the influence of inhomogeneities on the inflated envelopes of massive stars, as they occur when very massive stars (VMS) on the mainsequence, or evolved stars in the Wolf-Rayet (WR) phase, are approaching the Eddington limit.

In the following the current status of research on the inhomogeneities in stellar winds and envelopes is reviewed, and it is discussed how a connection of wind and envelope models may help to obtain more information on their uncertain clumping properties.

\section{The origin of clumping}

The presence of inhomogeneities has been predicted in a variety of theoretical studies of stellar winds and envelopes. In stellar winds, Doppler shifts introduced by velocity gradients lead to the so-called line-driven instability (Owocki et al. 1988), resulting in wind shocks. The shock-heated material can reach temperatures of several MK and is believed to be the origin of the observed X-Ray emission of single OB stars (Feldmeier et al. 1997). Further details on X-Ray wind diagnostics are discussed, e.g., by Oskinova (this volume).

Instabilities in stellar envelopes have been predicted by Glatzel (2008, 2009), for the non-linear regime of strange-mode pulsations (Saio et al. 1998; Glatzel \& Kaltschmidt 2002). The latter occur in cavities, which are typically formed in inflated stellar envelopes (cf. Sect.4.1). 3D models by Jiang et al. (2015) show that these systems can form a pronounced clumpy and porous structure (cf. also Jiang this volume).

Clumping and porosity have a strong effect on the mean opacity in astrophysical plasmas. There is a general trend that the increased densities within clumps, compared to a 
homogeneous medium, lead to an increase of the opacity (given in $\mathrm{cm}^{2} / \mathrm{g}$ ). Porosity, on the other hand, can lower the effective mean opacity, as, depending on the detailed clump geometry, velocity, and optical depth, radiation may leak through gaps between clumps. In the following we will discuss the effect of clumping and porosity on the diagnostics and the modelling of stellar winds and envelopes.

\section{Clumping in stellar winds}

\subsection{Wind diagnostics}

The empirical determination of stellar wind densities mainly relies on the analysis of optical recombination lines, such as $\mathrm{H} \alpha$. Because recombination is a two-particle process the emissivity of these lines scales with $\rho^{2}$, where $\rho$ denotes the enhanced clump density. The total line strength scales with the spatial mean $\left\langle\rho^{2}\right\rangle$. If we introduce a clumping factor such that the clump density $\rho=D \times\langle\rho\rangle$ and the inter-clump medium is void, the derived empirical mass-loss rates scale with $\dot{M} \propto 1 / \sqrt{D}$, where $D$ is usually ill-constrained. This introduces large uncertainties in empirically derived mass-loss rates. The scaling with $\dot{M} \propto 1 / \sqrt{D}$ is valid as long as individual clumps are optically thin in the diagnostic lines.

For an empirical determination of clumping factors it is necessary to compare empirical mass-loss rates based on $<\rho^{2}>$-diagnostics with values derived by different means. This was first done for WR stars, whose strong emission lines show electron-scattering wings whose strength scales linearly with $\langle\rho\rangle$ (Hillier 1984). Because of the presence of the electron-scattering wings, the clumping factors in WR winds are at least roughly known. The relative weakness of the electron-scattering wings typically implies clumping factors of the order of $D=10$ (cf. also Hamann \& Koesterke 1998), corresponding to mass-loss reductions of the order of 3 .

Fullerton et al. (2006) found a much higher mass-loss discrepancy of a factor 10 (corresponding to clumping factors of the order of $D=100$ ) for a large sample of Galactic O stars, based on the strength of PV P-Cygni absorption profiles obtained with the Far Ultraviolet Spectroscopic Explorer (FUSE). This extreme result implied that the wind mass-loss rates of $\mathrm{O}$ stars are much weaker than thought before, and started a broad discussion of the relevance of stellar-wind mass loss for massive stars (e.g. Smith \& Owocki 2006; Smith 2014). In the meantime it has become clear that the PV absorption profiles are also affected by porosity. This occurs when individual clumps become optically thick in the diagnostic lines, and radiation leaking through gaps between clumps affects the line profiles. Oskinova et al. (2007) pointed out that this results in much more moderate mass-loss reductions for $\mathrm{O}$ stars. The effects of porosity are further enhanced by discontinuities in the velocity fields of stellar winds (velocity-porosity; Owocki 2008).

While the inclusion of optically-thin clumping is now a standard in spectroscopic analyses, the effects of porosity have only been studied by combining standard non-LTE model atmospheres with 3D Monte-Carlo models (Sundqvist et al. 2010, 2011; Šurlan et al. 2012, 2013). These studies all suggest moderate mass-loss reductions. Sundqvist et al. (2014) pointed out that doublet ratios, i.e., the relative strength of different doublet components in UV resonance lines, can serve as a useful diagnostic means for clumping and porosity. In a homogeneous medium the doublet ratios should simply reflect the relative line strength of the components, as it follows from atomic physics. In a clumped and porous medium, also the clump geometry affects the line formation. For the most extreme case that individual clumps are optically thick and both doublet components 

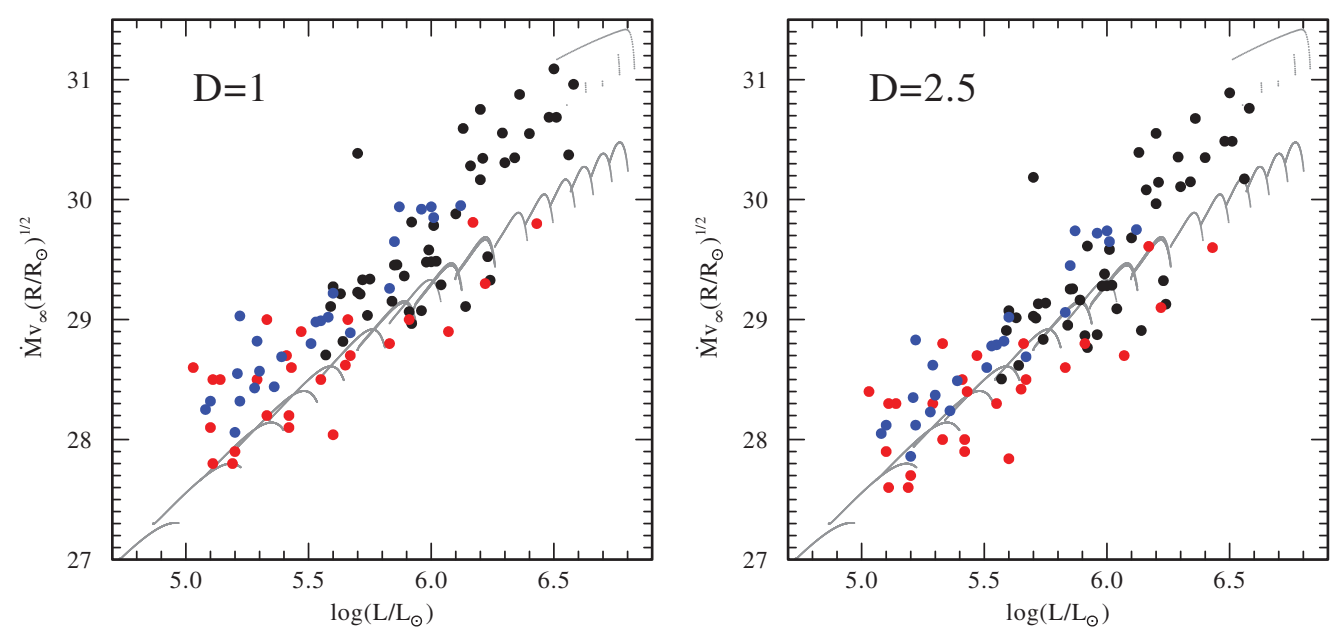

Figure 1. Wind momentum - luminosity relation for O-Of-WNh stars from the VLT-FLAMES Tarantula Survey. Filled circles indicate empirical results for O dwarfs (red), O giants and supergiants (blue), and the Of - WNh transition (black). The plot combines the results of three studies (see text). Shown are all results, regardless of sample overlaps. Upper limits, as they have been derived for some O-type stars with weak winds, have been skipped. The left panel shows the empirical results without clumping $(D=1)$. In the right panel the empirical results have been shifted according to an adopted clumping factor $D=2.5$. Grey lines indicate wind momenta for O-type stars that have been extracted from evolutionary tracks without rotation from Brott et al. (2011); Köhler et al. (2015).

are equally saturated, the line profiles are only affected by the clump geometry, and both doublet components appear equally strong.

The fact that the observed $\mathrm{P}_{\mathrm{V}}$ doublet ratios, in many cases, do not follow atomic physics is a direct sign of the existence of porosity, implying that the mass-loss reductions claimed by Fullerton et al. (2006) are too high. Sundqvist et al. (2014) further developed an effective opacity formalism for porous two-component media, and demonstrated that there are two solutions for the mass-loss rate, depending on whether an observed line profile is dominated by the clump, or inter-clump medium. This result likely resolves previous controversies about porous $\mathrm{O}$ star mass-loss rates from Monte-Carlo modeling.

The uncertainty in the clumping factors of $\mathrm{O}$ stars is still a major problem for the determination of empirical mass-loss rates. For this reason the clumping factor $D$ is usually treated as an open parameter. The best way forward in the understanding of clumping and porosity in stellar winds will be to include an appropriate effective opacity algorithm in non-LTE atmosphere models, and to perform detailed UV + optical studies of large stellar samples.

\subsection{Empirical mass-loss rates}

The most recent empirical results from optical analyses within the VLT-FLAMES Tarantula survey (Evans et al. 2011) are presented in Fig. 1. The plot shows results from three different studies of O dwarfs (Sabín-Sanjulián et al. 2017) in red, O giants and supergiants (Ramírez-Agudelo et al. 2017) in blue, and the Of - WNh transition regime of the most massive stars (Bestenlehner et al. 2014) in black. We compare the results with theoretical mass-loss rates that are extracted from stellar evolutionary models of Brott et al. (2011); Köhler et al. (2015), and which are based on the mass-loss estimates of Vink et al. (2000, 2001). First of all, ignoring the effects of clumping (left panel in Fig. 1), the 


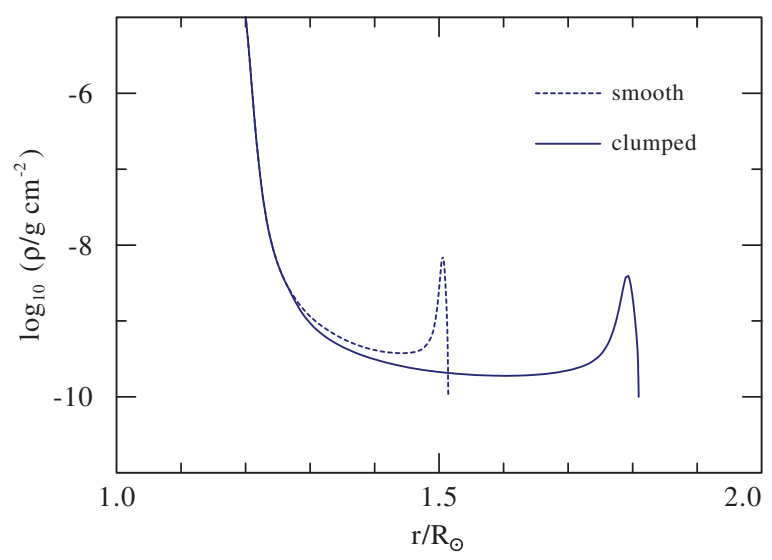

Figure 2. Density structure of inflated stellar envelopes with and without clumping from Gräfener et al. (2012). The clumped model is computed with a clumping factor $D=2$.

theoretical mass-loss rates match the empirical ones for $\mathrm{O}$ dwarfs, but are systematically lower than the ones for $\mathrm{O}$ giants/supergiants.

In the right panel of Fig. 1 a clumping factor of $D=2.5$ is adopted, introducing a shift of the empirical values by $\sqrt{D}$ so that the bulk of the $\mathrm{O}$ star data matches the theoretical mass-loss rates. This means that, if the real clumping factors are higher than 2.5, the mass-loss rates in stellar evolution models need to be corrected downwards by a factor $\sqrt{D / 2.5}$.

A second aspect in Fig. 1 are the enhanced mass-loss rates of VMS in the WNh phase. Wind models predict a strong dependence on the Eddington factor $\Gamma_{\mathrm{e}}$ in this regime Gräfener \& Hamann (2008), with a kink between optically thin O star winds and optically thick WNh stars (Vink et al. 2011). The strong dependence on $\Gamma_{\mathrm{e}}$ is supported by empirical studies of VMS in the Galactic center and the LMC (Gräfener et al. 2011; Bestenlehner et al. 2014). The WR mass-loss relations in evolutionary models (visible in the upper right corners in the diagrams in Fig. 1), which are based on the classical surface-chemistry criteria, fail to reproduce the observations. The extreme importance of VMS $\gtrsim 100 M_{\odot}$ for the ionising and mechanical feedback in young star-forming regions has been pointed out by Doran et al. (2013). A correct inclusion of these objects in evolutionary models and population synthesis computations thus seems to be crucial (cf. also Wofford et al. 2014; Gräfener \& Vink 2015; Crowther et al. 2016).

\section{Clumping in stellar envelopes}

Clumping in stellar envelopes is a relatively new topic that has been brought forward by Gräfener et al. (2012). Clumping turns out to be of major importance for inflated stellar envelopes because their structure is dominated by the topology of the Rosselandmean opacity in the stellar interior (given as a function of density $\rho$ and temperature $T$, or alternatively, gas pressure $P_{\text {gas }}$ and radiation pressure $\left.P_{\text {rad }}\right)$. In the following we will discuss how clumping and porosity can affect the envelope structure, and how stellar winds may connect to such envelopes.

\subsection{Stellar envelope inflation}

The envelope inflation effect is a radial inflation of the outer stellar envelope of stars near the Eddington limit (with classical Eddington factors of the order of 
$\Gamma_{\mathrm{e}}=\chi_{\mathrm{e}} L /(4 \pi c G M) \approx 0.5$, where $\chi_{\mathrm{e}}$ denotes the free-electron opacity). The effect has been studied with chemically homogeneous models for WR stars and massive stars on the ZAMS (Ishii et al. 1999; Petrovic et al. 2006; Gräfener et al. 2012; Ro \& Matzner 2016), and its relevance during the main-sequence evolution of massive stars at different metallicities has been discussed by Sanyal et al. $(2015,2017)$.

The underlying reason for the envelope inflation effect lies in the topology of the $\mathrm{Fe}$ opacity peak near $\sim 160 \mathrm{kK}$. As the envelope solution has to cross the region of the Fe opacity peak at some point, and the Fe opacity can become extremely high, the star struggles to avoid a super-Eddington situation in this region. In case that convection is efficient enough, this can be done by lowering the radiative flux in the region of high opacity. If convection is inefficient, the opacity $\chi(\rho, T)$ near the Fe-peak needs to be lowered. The only way to do this for given $T$, is to go to low densities $\rho$. Close to the Eddington limit, the topology of the opacity peak thus leads to the formation of a low-density region within the stellar envelope near $150 \mathrm{kK}$. Due to the relation between temperature and optical depth (approximately with $T^{4} \propto \tau$ ), and $\tau \propto \rho \Delta R$, a low density automatically implies a large radial extension $\Delta R$. Above the low-density zone the density increases again, so that a cavity is formed (cf. Fig. 2). Gräfener et al. (2012) described this effect in an analytical approach which revealed the existence of a stability limit, reminiscent of the S-Dor instability strip in the upper HRD. This makes the inflation effect one of the best candidates to explain the radius variations LBVs (cf. also Fig. 3).

Because of the importance of the density at the Fe opacity peak, the enhanced density in clumps has a major effect on the radii of stars in this regime. As long as individual clumps stay optically thin, clumping can be implemented in stellar models analogous to stellar atmosphere models, by simply computing the opacities $\chi(\rho, T)$ for a higher clump density $D \times \rho$. This approach has been used by Gräfener et al. (2012) to reproduce the observed radii of $\mathrm{H}$-free WR stars. The adopted clumping factors were of the order of $D=10$, and thus comparable to those found in WR-type stellar winds. This way it was possible to resolve the long-standing "WR radius problem" that the observed radii of WR stars are much larger (by up to a factor 10) than the ones predicted by classical stellar structure models (cf. Fig. 3).

As the inflation effect is expected to be highly dependent on metallicity (Ishii et al. 1999; Petrovic et al. 2006), it can also account for the fact that late WR subtypes are tendentially found in high-metallicity environments such as the Galactic center, while early subtypes dominate in low-metallicity environments such as the LMC. This is also supported by the study of McClelland \& Eldridge (2016) who used the same clumping approach to explain the properties of the WR populations in the Galaxy and LMC (see also McClelland this volume).

It is important to note that the research on the inflation effect is currently still in its infancy. The recent 3D simulations by Jiang et al. (2015) have shown that inflated envelopes may not only be clumped, but also porous. If clumps become optically thick in the continuum, the radiative flux tends to avoid high-density material, leading to an effective reduction of the Rosseland-mean opacity (cf. Shaviv 1998, 2001; Owocki et al. 2004). Furthermore, studies by Petrovic et al. (2006); Ro \& Matzner (2016) have shown that the dynamics of the envelope material in presence of a stellar wind can inhibit the inflation effect. To obtain a more realistic view on the inflation effect it will thus be necessary to include clumping and porosity in dynamic models stellar structure models.

\subsection{Connection with stellar winds}

Apart from purely dynamical effects, optically-thick stellar winds also impose boundary conditions on stellar envelopes because of their back warming. Gräfener \& Vink (2013) 


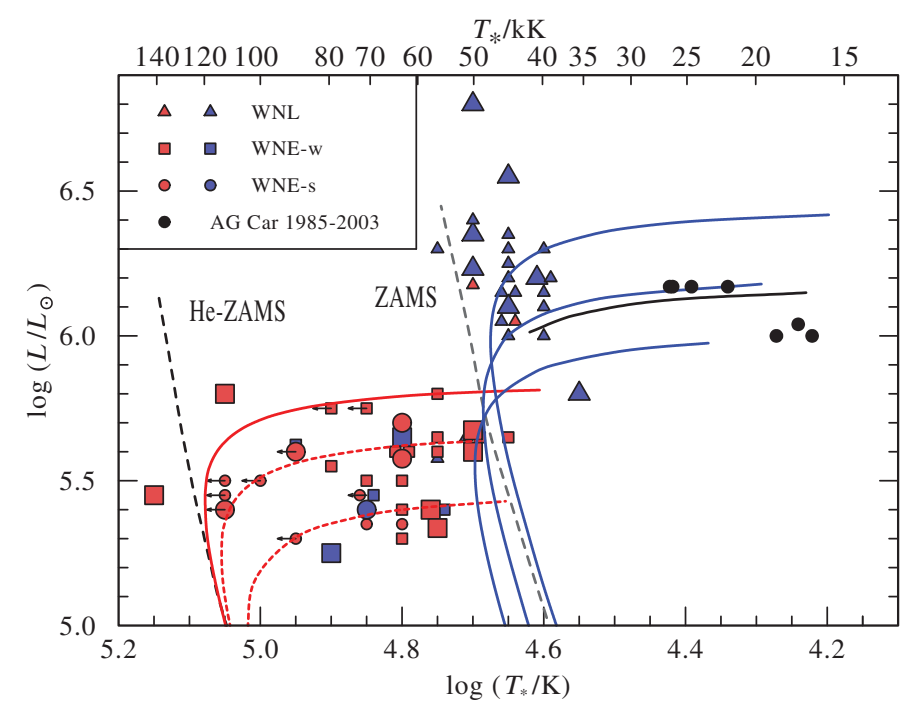

Figure 3. Hertzsprung-Russel diagram of the Galactic WN stars, and the LBV AGCar. Red/blue symbols indicate observed HRD positions of WN stars from Hamann et al. (2006) (blue: with hydrogen $(X>0.05)$; red: hydrogen-free $(X<0.05))$. Black symbols indicate the HRD positions of AG Car throughout its S Dor Cycle from 1985-2003, according to Groh et al. (2009). Large symbols refer to stars with known distances from cluster/association membership. The symbol shapes indicate the spectral subtype (see inlet). Arrows indicate lower limits of $T_{\star}$ for stars with strong mass loss. The observations are compared to stellar structure models from Gräfener et al. (2012) (blue with hydrogen, red hydrogen-free), and for AG Car ( $X=0.36$, black line). The dashed red lines indicate models for which clumping factors of 4 and 16 have been adopted to match the observed radii of $\mathrm{H}$-free WR stars.

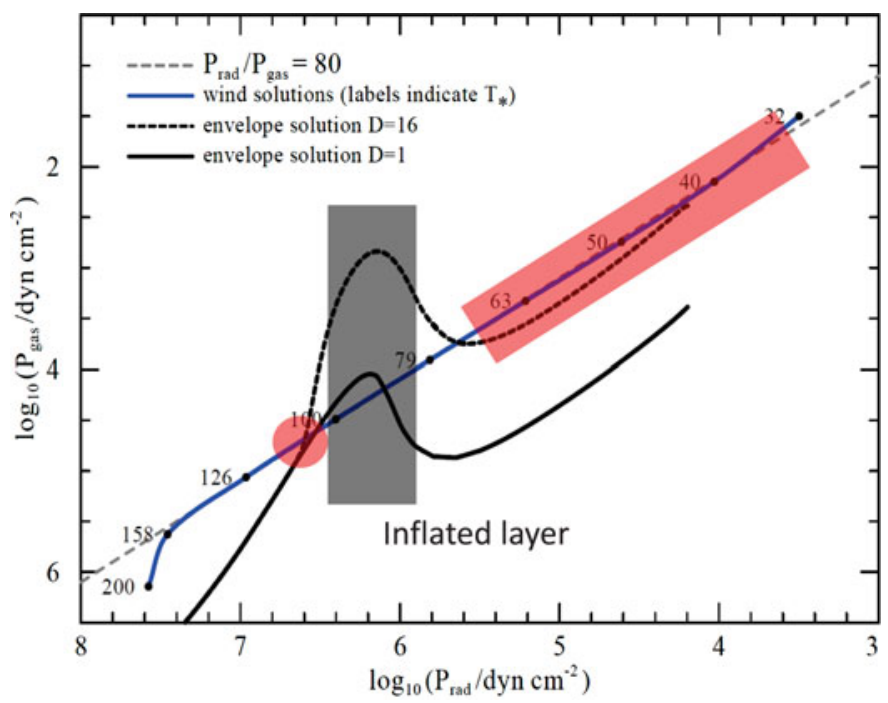

Figure 4. Conditions at the sonic point for a $14 M_{\odot}$ helium star with Galactic metallicity. The solid and dashed black lines indicate stellar envelope solutions with different clumping factors $D=1$ and 16 in the sub-surface layers. The conditions imposed by the optically thick stellar wind are indicated in blue. Labels indicate the stellar temperature $T_{\star}$ of the wind solutions. The red shaded areas indicate possible wind-envelope connections below (red circle) and above (red bar) the inflated envelope layers (grey). 
estimated the densities and temperatures at the sonic point of optically-thick radiativelydriven winds, and found that these correspond to very high, and almost constant ratios of $P_{\text {rad }} / P_{\text {gas }} \approx 80$. These high ratios reflect the ratio of the terminal wind velocity over the sonic speed $v_{\infty} / a$, and are independent of wind clumping. They are most likely the reason why optically-thick winds tend to occur only near the Eddington limit.

Fig. 4 demonstrates, for the example of a He star with $14 M_{\odot}$ and solar metallicity, that clumping can help to achieve a match between envelope and wind solutions at the sonic point. The reason is that clumping shifts the envelope solution to lower densities, and thus helps to achieve higher ratios of $P_{\mathrm{rad}} / P_{\text {gas }}$. For the un-clumped envelope solution (black solid curve in Fig. 4) a match between envelope and wind can only be achieved below the inflated layer (indicated as grey shaded area). For the clumped solution (dashed curve) such a match is also possible above the inflated zone. While these considerations are currently only of qualitative value, they show that the radii and mass-loss rates of stars with optically-thick winds may be the result of a very complex interplay between envelope, wind, and the clumping properties of the material in the envelope/wind transition region.

\section{Conclusions}

Clumping and porosity have strong influence on the effective opacities in stellar winds and envelopes. In particular, they affect the empirical determination of stellar wind massloss rates, and the radial extension of inflated stellar envelopes. A good knowledge of clumping and porosity is thus crucial for the understanding of the mass loss, and the radii of massive stars.

The current lack of accurate knowledge of clumping factors and porosity demand for the inclusion of new approaches for the description of clumpy and porous media in stellar atmosphere and stellar structure models, and their exposition to empirical tests.

\section{References}

Bestenlehner, J. M., Gräfener, G., Vink, J. S., et al. 2014, A\&3A 570, A38

Brott, I., de Mink, S. E., Cantiello, M., et al. 2011, A\&A 530, A115

Crowther, P. A., Caballero-Nieves, S. M., Bostroem, K. A., et al. 2016, MNRAS 458, 624

Doran, E. I., Crowther, P. A., de Koter, A., et al. 2013, A\& A 558, A134

Evans, C. J., Taylor, W. D., Hénault-Brunet, V., et al. 2011, A\&A 530, A108

Feldmeier, A., Kudritzki, R.-P., Palsa, R., Pauldrach, A. W. A., \& Puls, J. 1997, A\& A 320, 899

Fullerton, A. W., Massa, D. L., \& Prinja, R. K. 2006, ApJ 637, 1025

Glatzel, W. 2008, in A. Werner \& T. Rauch (ed.), Hydrogen-deficient stars, Vol. 391 of Astronomical Society of the Pacific Conference Series, p. 307, San Francisco: Astronomical Society of the Pacific

Glatzel, W. 2009, Communications in Asteroseismology 158, 252

Glatzel, W. \& Kaltschmidt, H. O. 2002, MNRAS 337, 743

Gräfener, G. \& Hamann, W.-R. 2008, A\& A 482, 945

Gräfener, G., Owocki, S. P., \& Vink, J. S. 2012, A\&\&A 538, A40

Gräfener, G. \& Vink, J. S. 2013, A\&A 560, A6

Gräfener, G. \& Vink, J. S. 2015, A\&A 578, L2

Gräfener, G., Vink, J. S., de Koter, A., \& Langer, N. 2011, A\&A 535, A56

Groh, J. H., Hillier, D. J., Damineli, A., et al. 2009, ApJ 698, 1698

Hamann, W.-R., Gräfener, G., \& Liermann, A. 2006, A $\& A$ 457, 1015

Hamann, W.-R. \& Koesterke, L. 1998, A\& A 335, 1003

Hillier, D. J. 1984, ApJ 280, 744

Ishii, M., Ueno, M., \& Kato, M. 1999, PASJ 51, 417 
Jiang, Y.-F., Cantiello, M., Bildsten, L., Quataert, E., \& Blaes, O. 2015, ApJ 813, 74

Köhler, K., Langer, N., de Koter, A., et al. 2015, A\&̊A 573, A71

McClelland, L. A. S. \& Eldridge, J. J. 2016, MNRAS 459, 1505

Oskinova, L. M., Hamann, W.-R., \& Feldmeier, A. 2007, A\& A 476, 1331

Owocki, S. P. 2008, in W.-R. Hamann, A. Feldmeier, \& L. M. Oskinova (eds.), Clumping in Hot-Star Winds, p. 121

Owocki, S. P., Castor, J. I., \& Rybicki, G. B. 1988, ApJ 335, 914

Owocki, S. P., Gayley, K. G., \& Shaviv, N. J. 2004, ApJ 616, 525

Petrovic, J., Pols, O., \& Langer, N. 2006, A\&A 450, 219

Ramírez-Agudelo, O. H., Sana, H., de Koter, A., et al. 2017, ArXiv e-prints 1701.04758

Ro, S. \& Matzner, C. D. 2016, ApJ 821, 109

Sabín-Sanjulián, C., Simón-Díaz, S., Herrero, A., et al. 2017, ArXiv e-prints 1702.04773

Saio, H., Baker, N. H., \& Gautschy, A. 1998, MNRAS 294, 622

Sanyal, D., Grassitelli, L., Langer, N., \& Bestenlehner, J. M. 2015, A\&A 580, A20

Sanyal, D., Langer, N., Szécsi, D., -C Yoon, S., \& Grassitelli, L. 2017, A $8 A$ 597, A71

Shaviv, N. J. 1998, ApJ 494, L193

Shaviv, N. J. 2001, ApJ 549, 1093

Smith, N. 2014, ARAA 52, 487

Smith, N. \& Owocki, S. P. 2006, ApJ 645, L45

Sundqvist, J. O., Puls, J., \& Feldmeier, A. 2010, A\&A 510, A11

Sundqvist, J. O., Puls, J., Feldmeier, A., \& Owocki, S. P. 2011, A\&A 528, A64

Sundqvist, J. O., Puls, J., \& Owocki, S. P. 2014, A\&A 568, A59

Šurlan, B., Hamann, W.-R., Aret, A., et al. 2013, A\&A 559, A130

Šurlan, B., Hamann, W.-R., Kubát, J., Oskinova, L. M., \& Feldmeier, A. 2012, A\&\&A 541, A37

Vink, J. S., de Koter, A., \& Lamers, H. J. G. L. M. 2000, A\&A 362, 295

Vink, J. S., de Koter, A., \& Lamers, H. J. G. L. M. 2001, $A \& B A 369,574$

Vink, J. S., Muijres, L. E., Anthonisse, B., et al. 2011, A\& $A$ 531, A132

Wofford, A., Leitherer, C., Chandar, R., \& Bouret, J.-C. 2014, ApJ 781, 122 